\title{
Influenza-associated paediatric hospitalisations in seasons 2011-2012 to 2016-2017 in the Valencia Hospital Network for the Study of Influenza
} and Other Respiratory Viruses (Spain)

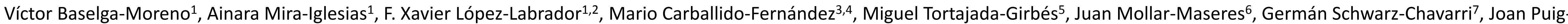
Barberà ${ }^{1,8}$, Javier Díez-Domingo ${ }^{1}$ for the Valencia Hospital Network for the Study of Influenza and Other Respiratory Viruses (VAHNSI)

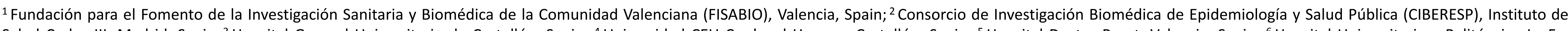

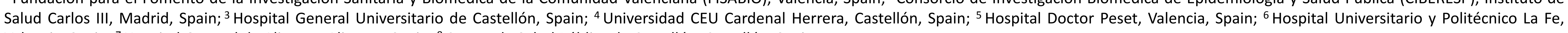
Valencia, Spain; ${ }^{7}$ Hospital General de Alicante, Alicante, Spain; ${ }^{8}$ Centro de Salud Pública de Castellón, Castellón, Spain

\section{Introduction}

The Valencia Hospital Network for the Study of Influenza and Other Respiratory Viruses (VAHNSI) aims to determine the burden of severe influenza disease and influenza vaccine effectiveness. In this study, our objective will be to determine the incidence of influenza in hospitalisations in children and patients characteristics in Valencia Region, Spain
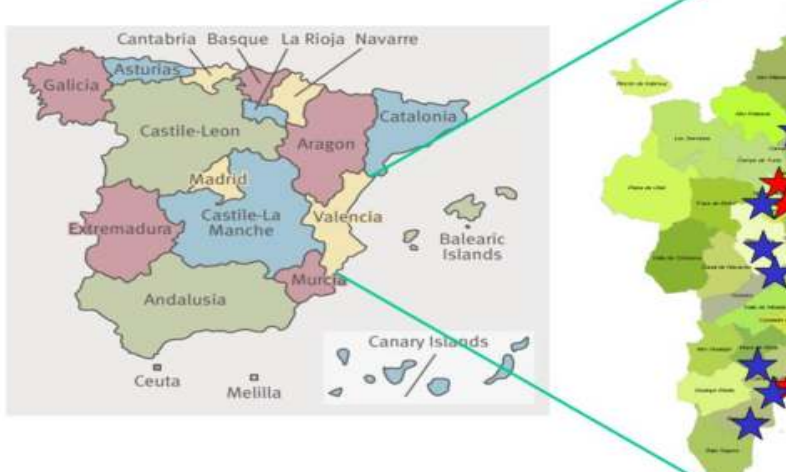

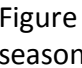

Methods

This study was based on a prospective active surveillance in 4-10 hospitals, during 6 seasons (November-April). Subjects under 18 years not institutionalised, with symptoms compatible with influenza were approached, and included after informed consent signature. Patients hospitalised in previous 30 days, or those for which symptoms appeared 8 days or more before being hospitalised were excluded Nasopharyngeal and pharyngeal or nasal swabs were collected, and patients were tested for influenza by real-time reverse transcriptionpolymerase chain reaction (RT-PCR).

Results

Among the 6332 screened patients, 4803 were eligible. 265 did not sign the consent. Therefore, 4538 patients were included, and 4517 of them were tested for influenza. Among them, 2322 (51.41\%) were less than one year old, 1717 (38.01\%) were 1 to 4 years old, and 478 (10.58\%) were 5 to 17 years old.
Globally, 102 patients tested positive by influenza A(H1N1)pdm09, 169 patients tested positive by influenza $A(H 3 N 2), 24$ had influenza $A$ not subtyped and 62 had influenza $B$.

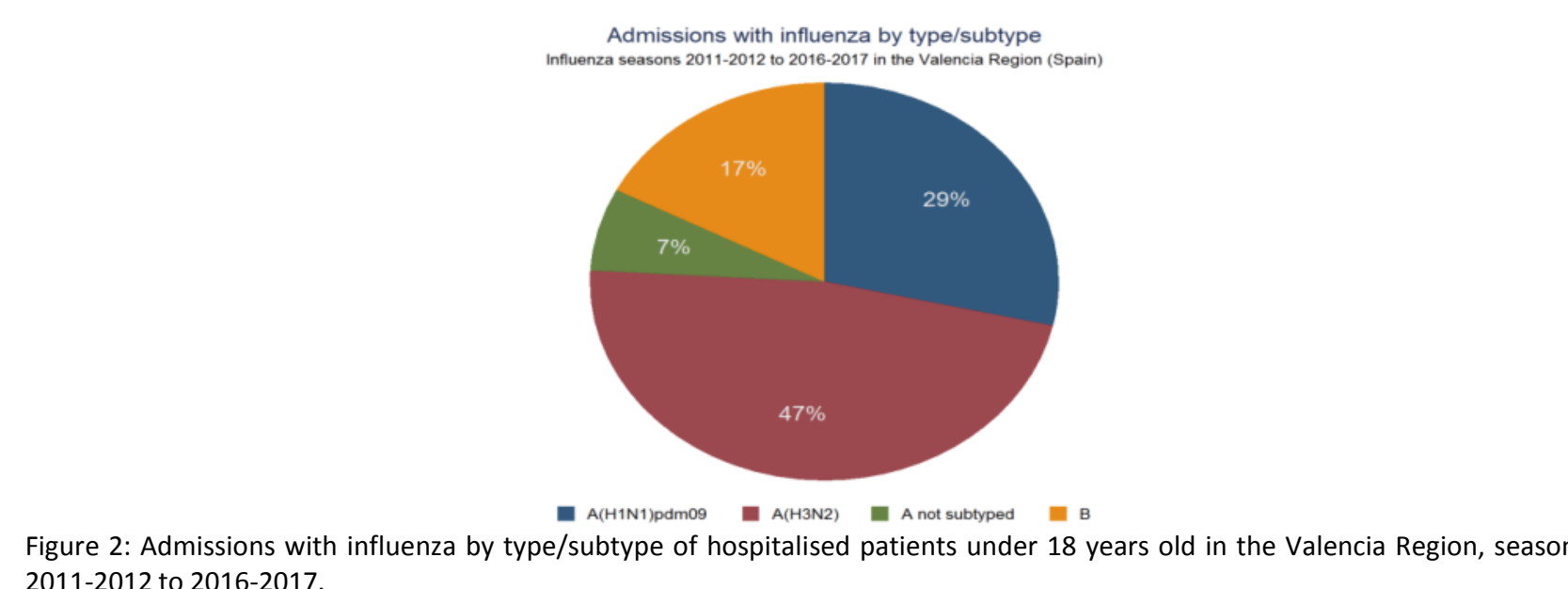
Figure 2: Admissions with
2011-2012 to 2016-2017

Although the majority of the patients included were less than one year old, the highest number of influenza cases was found in those from 1 to 4 years old, with 174 influenza cases (10.13\% among tested patients in this age group), and the highest percentage of influenza cases was found in patients 5 to 17 years old (60 cases with influenza among 478 patients tested, $12.55 \%$ ), whereas in those under one year, there were 123 influenza cases (5.30\% among tested patients in this age group) Influenza A was found in $89 \%$ of the cases in patients under 5 years old, and Influenza B was predominant in the age group 5-17 years old, with 30 of 60 influenza cases. In the other age groups, and also globally, the predominant strain was the $A(H 3 N 2)$, followed by $A(H 1 N 1) p d m 09$.

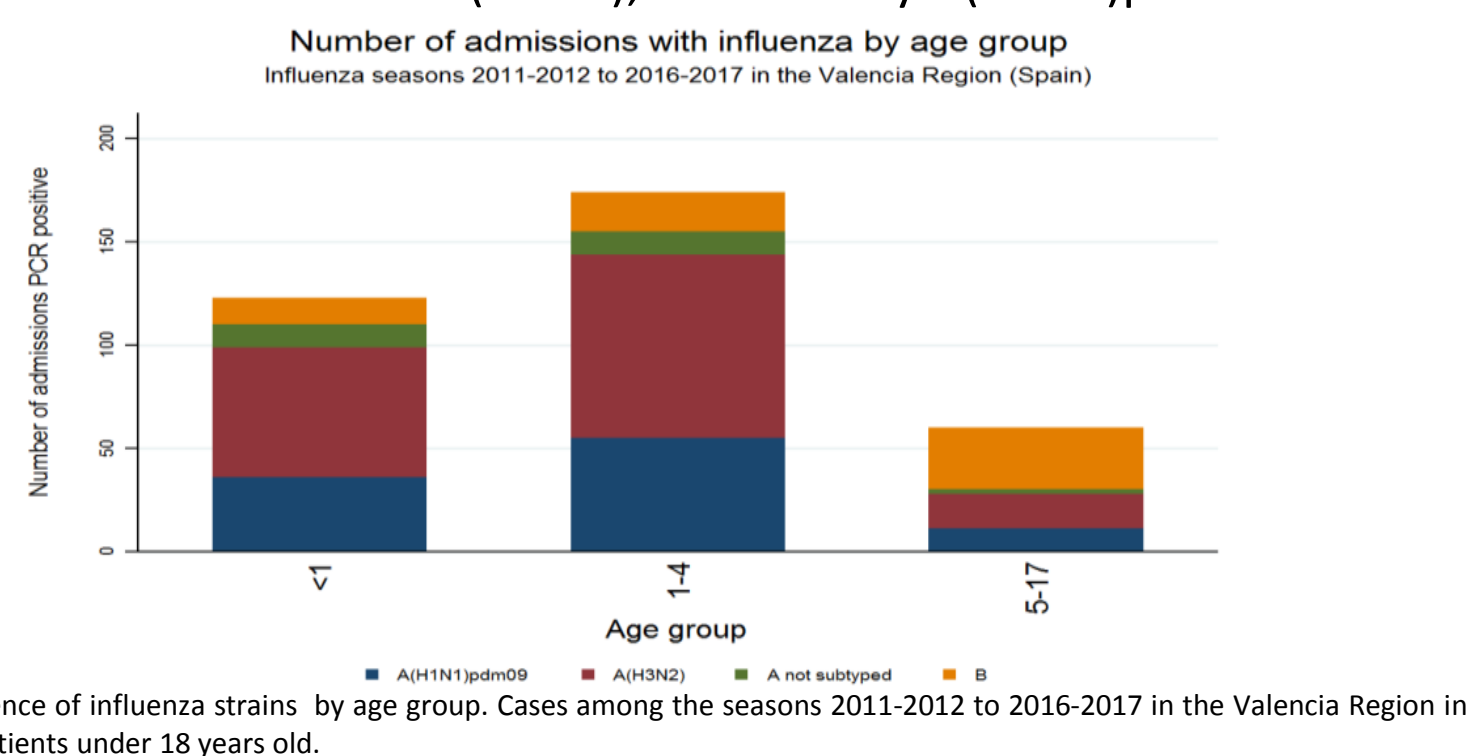

Influenza $\mathrm{A}(\mathrm{H} 3 \mathrm{~N} 2)$ was predominant in 3 seasons, whereas influenza $B$ was predominant in 2 seasons and $A(\mathrm{H} 1 \mathrm{~N} 1)$ pdm09 was predominant only in one season.
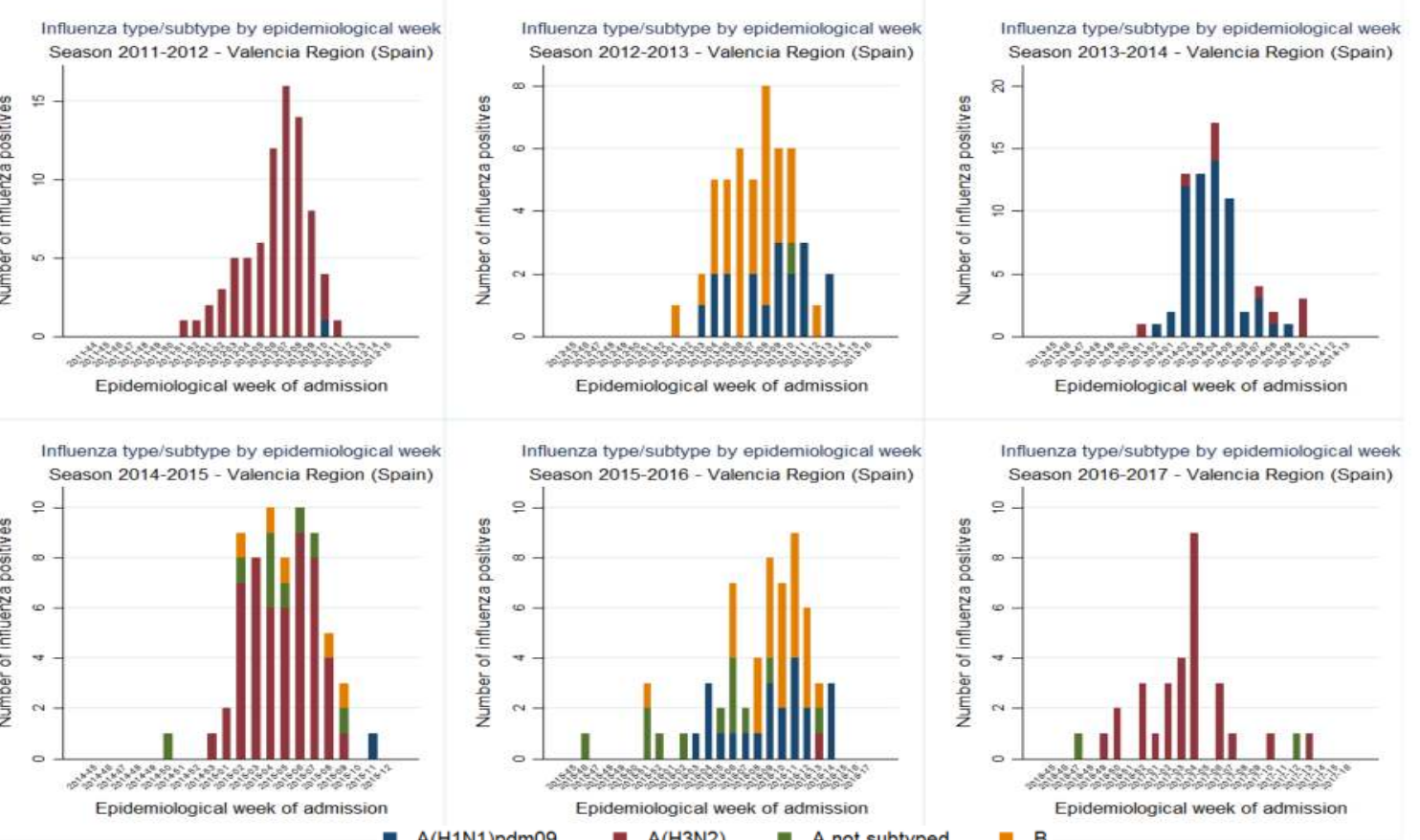

Figure 4: Admissions with influenza by epidemiological we
the Valencia Region, seasons $2011-2012$ to $2016-2017$ Among the 4538 patients included, 204 (4.50\%) were vaccinated. Vaccination coverage was $9.40 \%(50 / 532)$ in children with asthma or bronchitis.

Length of hospitalisations was similar among influenza positives and negatives, with a mean of $5.03(95 \% \mathrm{Cl}(4.88,5.18))$ in less than one year, $4.14(95 \% \mathrm{Cl}(3.97,4.30))$ in $1-4$ years, and $4.24(95 \% \mathrm{Cl}(3.92,4.56))$ in patients $5-17$ years.

\section{Conclusions}

Influenza had a moderate impact in children. Influenza B represented $17.37 \%$ of the influenza cases in these 6 seasons. Influenza $B$ had similar incidence compared with influenza $A$ among influenza positives 5 to 17 years old. Influenza A was predominant in patients under 5 years old. There was low vaccination coverage in children, even in those with asthma or bronchitis, where vaccination is recommended and free of charge. 